\title{
Frequent epigenetic inactivation of RASSF2 in thyroid cancer and functional consequences
}

\author{
Undraga Schagdarsurengin ${ }^{1 \dagger}$, Antje M Richter ${ }^{1 \dagger}$, Juliane Hornung ${ }^{1,2 \dagger}$, Cornelia Lange $^{1 \dagger}$, Katrin Steinmann $^{1}$, \\ Reinhard H Dammann ${ }^{*}$
}

\begin{abstract}
Background: The Ras association domain family (RASSF) encodes for distinct tumor suppressors and several members are frequently silenced in human cancer. In our study, we analyzed the role of RASSF2, RASSF3, RASSF4, RASSF5A, RASSF5C and RASSF6 and the effectors MST1, MST2 and WW45 in thyroid carcinogenesis.

Results: Frequent methylation of the RASSF2 and RASSF5A CpG island promoters in thyroid tumors was observed. RASSF2 was methylated in $88 \%$ of thyroid cancer cell lines and in $63 \%$ of primary thyroid carcinomas. RASSF2 methylation was significantly increased in primary thyroid carcinoma compared to normal thyroid, goiter and follicular adenoma $(0 \%, 17 \%$ and $0 \%$, respectively; $p<0.05)$. Patients which were older than 60 years were significantly hypermethylated for RASSF2 in their primary thyroid tumors compared to those younger than 40 years (90\% vs. 38\%; $p<0.05$ ). RASSF2 promoter hypermethylation correlated with its reduced expression and treatment with a DNA methylation inhibitor reactivated RASSF2 transcription. Over-expression of RASSF2 reduced colony formation of thyroid cancer cells. Functionally our data show that RASSF2 interacts with the proapoptotic kinases MST1 and MST2 and induces apoptosis in thyroid cancer cell lines. Deletion of the MST interaction domain of RASSF2 reduced apoptosis significantly $(p<0.05)$.
\end{abstract}

Conclusion: These results suggest that RASSF2 encodes a novel epigenetically inactivated candidate tumor suppressor gene in thyroid carcinogenesis.

\section{Background}

Approximately $10 \%$ of the whole population develop a clinically significant thyroid nodule during their lifetime [1]. Nearly $80 \%$ of all thyroid malignancies arise from the follicular epithelial cells, the thyrocytes. There are three main histological types of epithelial thyroid cancer: the papillary thyroid carcinoma (PTC), the follicular thyroid carcinoma (FTC) and the undifferentiated (or anaplastic) thyroid carcinoma (UTC). In contrast, medullary thyroid carcinoma (MTC) develop from parafollicular C cells. $\mathrm{C}$ cells produce calcitonin, a hormone regulating the calcium metabolism [2].

Development and progression of thyroid tumors are a consequence of complex epigenetic and genetic changes. In our previous work, we have identified a novel Ras effector, which is located at the tumor suppressor

\footnotetext{
* Correspondence: reinhard.dammann@gen.bio.uni-giessen.de † Contributed equally

${ }^{1}$ Institute for Genetics, Justus-Liebig-University Giessen, Germany Full list of author information is available at the end of the article
}

gene locus 3p21.3 [3]. The gene termed Ras Association Domain Family 1A (RASSF1A) is epigenetically inactivated in human cancers and inhibits the growth of tumors in vivo $[4,5]$. We and others have shown that RASSF1A is frequently silenced in thyroid cancer [6-9]. RASSF1A hypermethylation was more pronounced in the aggressive undifferentiated thyroid carcinoma $[7,10]$. Epigenetic inactivation of RASSF5 in thyroid cancer has also been investigated $[11,12]$.

RASSF1A was the first member of the Ras association domain family (RASSF). The family consists of six classical RASSFs (RASSF1 to RASSF6) and the N-terminal RASSFs (RASSF7 to RASSF10) with a N-terminal Ras association (RA) domain [13-17]. The classical RASSFs encode a C-terminal RA and SARAH domain $[13,16]$. Several of these genes (e.g. RASSF1A, RASSF2, RASSF5 [NORE1], RASSF6) encode tumor suppressors, which are involved in cell cycle regulation, microtubule stability and in apoptosis $[13,16,18-20]$. The SARAH domain is a protein-protein interaction domain, named after the

\section{Biomed Central}


tumor suppressors Salvador (in D. melanogaster; orthologue of human WW45), RASSF1 and Hippo (in D. melanogaster; orthologue of the human proapoptotic kinase MST1). SARAH domains are found in WW45, MST1 and all classical RASSFs. Heteromeric as well as homomeric interactions can be conducted via their SARAH domains [21], e.g. MST and WW45 or RASSF1A and RASSF5 interaction [22-24]. SARAH domains play a central role in the newly discovered Hippo signaling pathway in D. melanogaster, which regulates cell proliferation and apoptosis [21,25].

The aim of our study was to reveal the epigenetic status and function of RASSF2, RASSF3, RASSF4, RASSF5 and RASSF6 in thyroid carcinogenesis. Here, we report that RASSF2 is frequently hypermethylated in thyroid tumors and suppresses growth of thyroid cancer.

\section{Results \\ Epigenetic inactivation of RASSF2 and RASSF5 in thyroid cancer}

In our study we aimed to reveal the role of the classical RASSF members which harbor a C-terminal RA domain and a SARAH domain and their effectors MST1, MST2 and WW45 in thyroid carcinogenesis. Since RASSF1A function and silencing was already studied in detail, we focused our work on RASSF2, RASSF3, RASSF4, RASSF5A, RASSF5C and RASSF6 which all encode a Cterminal SARAH domain $[7,10,13]$. First we analyzed the promoter methylation of these genes in thyroid cancer cell lines (Fig. 1). RASSF6 contains the only promoter, which is not located within a CpG island [13] and was not included in the methylation analysis. RASSF 2 promoter hypermethyation was observed in seven out of eight (88\%) thyroid cancer cell lines (Fig. 1 and Tab. 1). RASSF5A hypermethylation was found in all analyzed cancer cell lines, however $R A S S F 5 C$ was only partially methylated in $8305 \mathrm{C}$ and Hth74 (Fig. 1). RASSF5A and $R A S S F 5 C$ are transcribed from different CpG island promoters [13]. CpG island hypermethylation of RASSF2 and $R A S S F 5 A$ was confirmed by bisulfite sequencing (additional file 1). CpG island promoter hypermethylation of RASSF3, RASSF4, MST1, MST2 and WW45 were not found in thyroid cancer cell lines (Fig. 1). RASSF1A methylation was analyzed previously and is summarized in Table $1[7,10]$. RASSF1A was hypermethylated in all analyzed thyroid cancer cell lines.

Next we analyzed the expression of RASSF2, RASSF5A and RASSF5C in all thyroid cancer cell lines and human fibroblasts (HF53) (Fig. 2). In human fibroblasts, which were unmethylated for all three promoters, high expression of RASSF 2 and RASSF5A was detected, however RASSF5C expression was low (Fig. 2A). Expression of RASSF2 was reduced in several thyroid cancer cell lines (e.g. FTC 236 and 1736). RASSF5A expression was also diminished in different thyroid cancer cell lines (Fig. 2A) compared to HF53. Additionally, two thyroid cancer cell lines (FTC 236 and 1736) were treated with 5-Aza-2'-deoxycytidine (Aza) and RASSF2 and RASSF5A expression was analyzed by qRT-PCR (Fig. 2B and 2C). Inhibition of DNA methyltransferases by Aza induced an upregulation of RASSF 2 and RASSF5A expression. In summary, we observed frequent epigenetic silencing of RASSF 2 and RASSF5A in thyroid cancer cell lines.

\section{Frequent tumor-specific methylation of RASSF2 in primary thyroid tumors}

Subsequently, we analyzed the methylation of RASSF2 and RASSF5A promoter in 31 primary thyroid carcinomas (3 MTC, 10 FTC, 12 PTC and 6 UTC), 10 follicular adenomas, 12 goiters and 12 normal thyroid controls (Fig. 3 and Tab. 1). In 19 out of 30 (63\%) thyroid carcinomas, the promoter of RASSF2 was partially methylated (Tab. 1). In contrast, none of 12 normal thyroid tissues and 10 follicular adenomas exhibited a methylation of RASSF2 (Fig. 3A). Only two out of 12 (17\%) goiters were methylated (Tab. 1). Statistical analysis revealed a significant increase in RASSF2 methylation frequency in thyroid cancer compared to normal thyroid controls, follicular adenomas or goiters $(\mathrm{p}<0.001, \mathrm{p}=$ 0.001 or $\mathrm{p}=0.015$; Fisher's exact test, respectively). Furthermore, we examined the methylation frequency of RASSF2 in different types of thyroid carcinoma (Tab. 1). Medullary thyroid carcinoma showed the lowest frequency $(0 / 3=0 \%)$ in comparison to PTC $(6 / 11=54 \%)$, to FTC $(8 / 10=80 \%)$ and to UTC $(5 / 6=83 \%)$. Methylation of RASSF2 was significantly higher in thyroid tumors obtained from older patients (90\%; $>60$ year) compared to those from younger patients $(38 \% ;<40$ years; $\mathrm{p}=0.043$ ) or patients from 41 to 59 years $(58 \%)$. Other correlations with histopathological parameters were not revealed. We also analyzed the RASSF5A methylation in primary thyroid cancers and other thyroid tissues (Fig. 3B). RASSF5A methylation was frequently found in cancer cell lines $(9 / 9=100 \%)$ and primary thyroid cancer but was also detected in all analyzed control tissues, goiters and follicular adenomas (Tab. 1). Since RASSF5A methylation was not tumor specific, we further focused on RASSF2 where methylation was preferentially found in cancers.

\section{Functional analysis of RASSF2}

Next, we analyzed the distinct tumor suppressive features associated with RASSF2. First, the localization of RASSF2 and the other classical RASSFs was investigated in FTC133 cells (Fig. 4). RASSF1A and RASSF1C are both found in the cytoplasm together with microtubule. The other RASSFs (RASSF2, Rassf3, RASSF4, RASSF5 and RASSF6) were not associated with microtubule but 


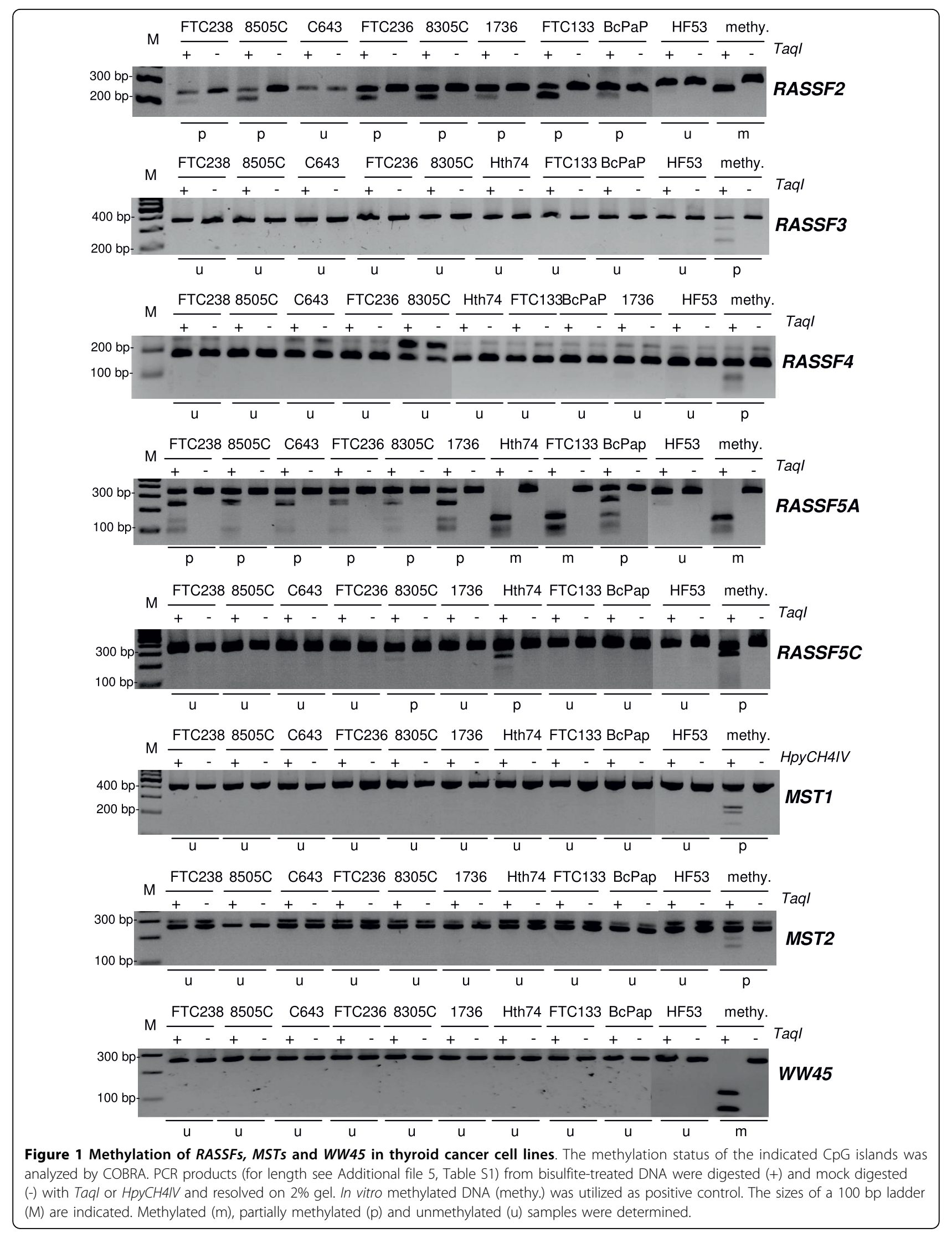


Table 1 Summary of RASSF2, RASSF5A and RASSF1A methylation analyses

\begin{tabular}{clll}
\hline & RASSF2 & RASSF5A & RASSF1A $^{\mathbf{c}}$ \\
\hline thyroid cancer cell lines & $7 / 8(88 \%)$ & $9 / 9(100 \%)$ & $9 / 9(100 \%)$ \\
\hline primary thyroid cancer & $19 / 30(63 \%)^{\mathrm{a}, ~ b}$ & $26 / 31(84 \%)$ & $26 / 31(84 \%)$ \\
MTC & $0 / 3(0 \%)$ & $1 / 3(33 \%)$ & $5 / 6(83 \%)$ \\
PTC & $6 / 11(54 \%)$ & $9 / 12(75 \%)$ & $8 / 13(62 \%)$ \\
FTC & $8 / 10(80 \%)$ & $10 / 10(100 \%)$ & $7 / 10(70 \%)$ \\
UTC & $5 / 6(83 \%)$ & $6 / 6(100 \%)$ & $7 / 9(77 \%)$ \\
\hline follicular adenoma & $0 / 10(0 \%)^{a}$ & $10 / 10(100 \%)$ & $7 / 10(70 \%)$ \\
\hline goiter & $2 / 12(17 \%)^{b}$ & $12 / 12(100 \%)$ & $9 / 12(75 \%)$ \\
\hline control tissue & $0 / 12(0 \%)^{a}$ & $12 / 12(100 \%)$ & - \\
\hline
\end{tabular}

significant differences: ${ }^{a}$ primary thyroid carcinoma vs. follicular adenoma or primary thyroid carcinoma vs. normal thyroid $\left(p \leq 0.001\right.$, Fisher's exact test); ${ }^{b}$ primary thyroid carcinoma vs. goiter $\left(p=0.015\right.$, Fisher's exact test) ${ }^{\mathrm{C}}[7,10]$. were observed in the cytoplasm and nucleus (Fig. 4 and data not shown). For Rassf3, RASSF4, RASSF5 and RASSF6 cluster formation was detected (Fig. 4 and data not shown). Deletion of the SARAH domain did not alter the localization of the fluorescent RASSF2, Rassf3 and RASSF6. However RASSF1A and RASSF4 appear to localize to nucleus from cytoplasm. It is interesting to note that the utilized RASSF5A isoform (variant 4) contains a partially truncated SARAH domain. The SARAH domain is a protein-protein interaction domain, which is found in all classical RASSFs and their proapoptotic effector MST1 and MST2 and the adapter protein WW45. Therefore, we analyzed the interaction of these proteins in yeast two hybrid and pull down assays (Table 2 and Fig. 5). We found an interaction of all

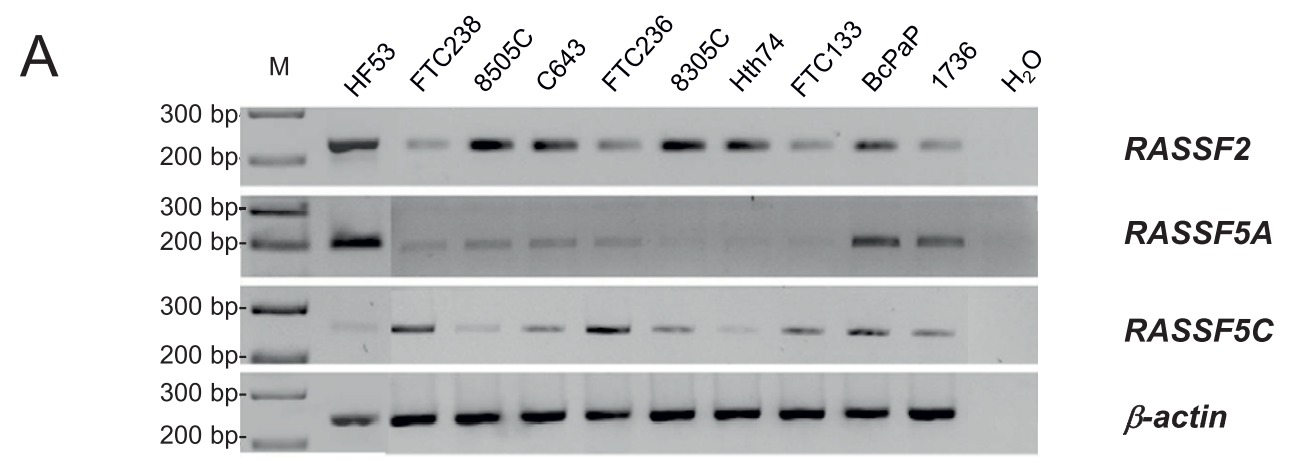

$\mathrm{B}$

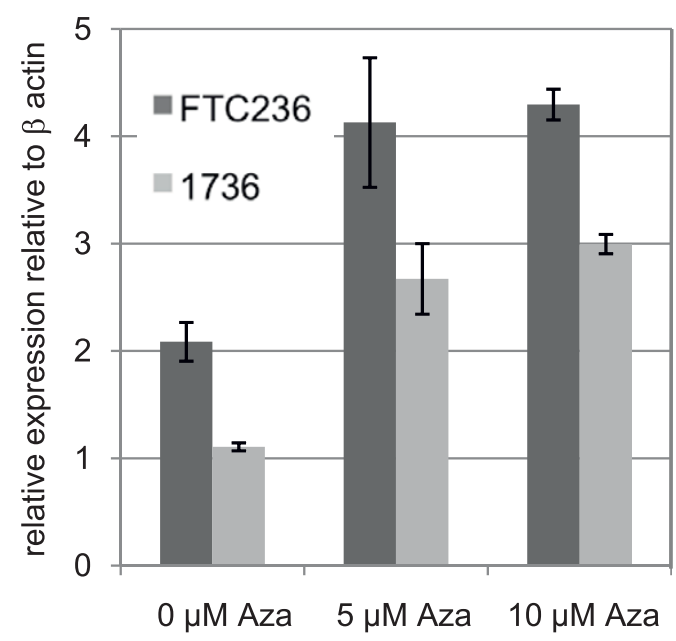

C

RASSF5A

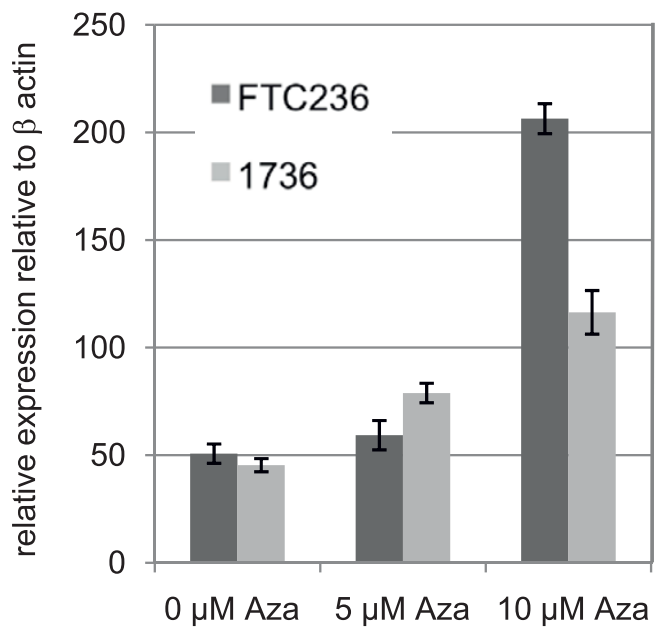

Figure 2 RASSF2 and RASSF5 expression in thyroid cancer cell lines. cDNA isolated from thyroid cancer cell lines and human fibroblasts (HF53) were amplified by RT-PCR (for primer and conditions see Additional file 6, Table $\mathrm{S} 2$ ) together with a water control $\left(\mathrm{H}_{2} \mathrm{O}\right)$. PCR products were resolved on $2 \%$ gel together with a $100 \mathrm{bp}$ ladder (M). Expression of $\beta$-actin (230 bp) was determined as a control for RNA integrity. B. Reexpression of RASSF2 was analyzed by qRTPCR in thyroid cancer cell lines FTC133 and 1736 treated for 4 days with indicated concentrations of 5-aza-2'-deoxycytidine (Aza). RNA levels were normalized to $\beta$-actin and calculated relative to human fibroblasts (= 100) $\mathbf{C}$. Reexpression of RASSF5A after Aza treatment. 


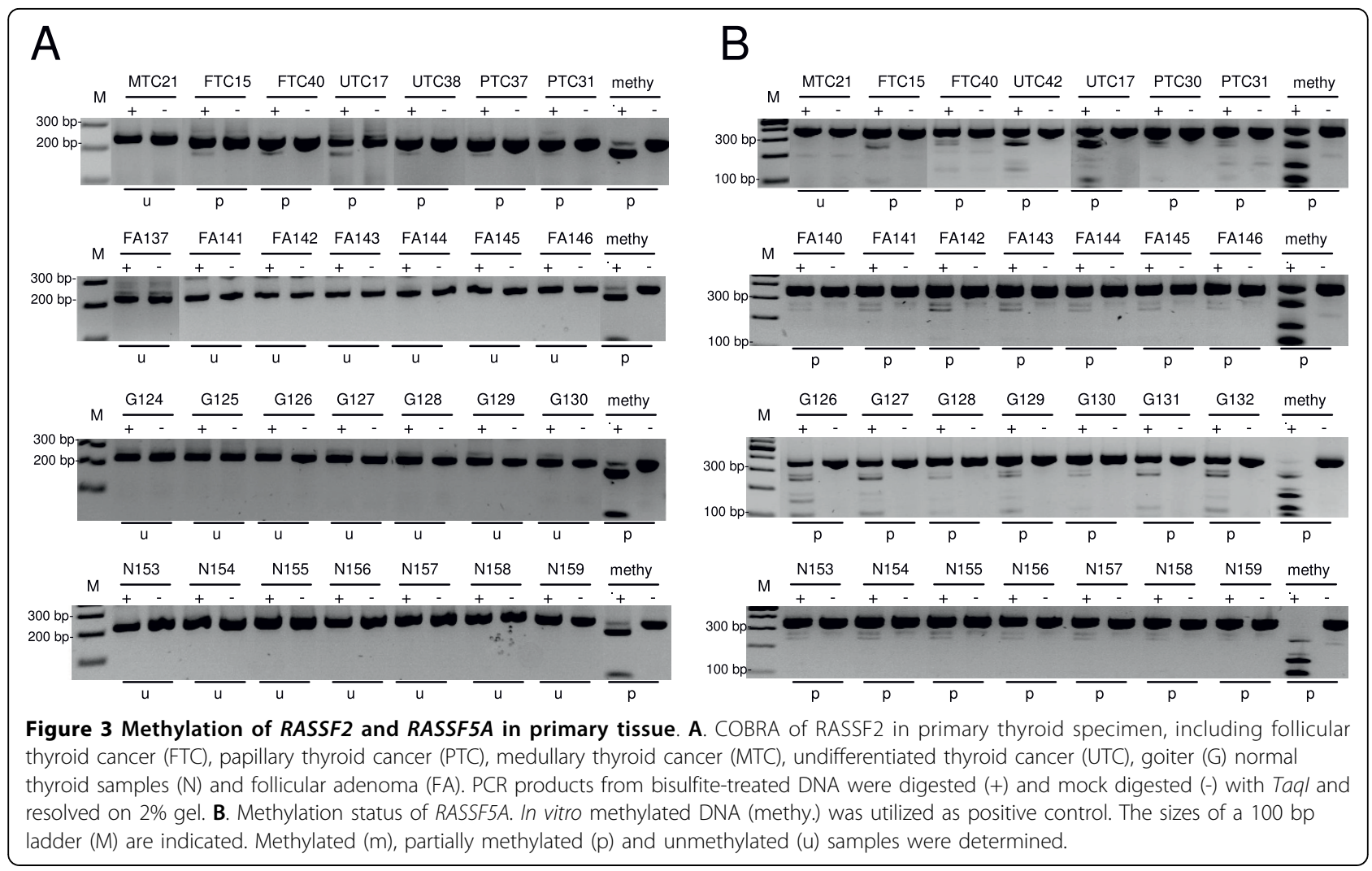

RASSFs with MST1 and MST2 in the yeast two hybrid assay (additional file 2). However, truncation of the SARAH of RASSF5A (variant 4) and RASSF2 reduced the interaction in the yeast two hybrid or abolished interaction in the pulldown assay (Fig. 5, additional file 2 and additional file 3). Interaction of WW45 with RASSF1A, RASSF3, RASSF4 and MST1 and MST2 in the yeast two hybrid was found. Precipitation of MST1 with WW45, RASSF1A, RASSF2, RASSF3, RASSF5C and RASSF6 was also confirmed by pull down (Tab. 2 and Fig. 5). Interaction of RASSF2 with RASSF1A, RASSF3 and RASSF5C was only found in yeast two hybrid assay (Tab. 2).

\section{RASSF2-induced growth suppression and apoptosis in thyroid cancer}

The interaction of RASSF2 with kinases MST1 and MST2 indicate that RASSF2 is involved in the proapoptotic RASSF-pathway. Therefore, we analyzed RASSF2induced growth suppression and apoptosis in thyroid cancer cell lines (Fig. 6). The thyroid cancer cell line 1736 was transfected with a construct expressing RASSF2 under control of the CMV promoter or an empty vector (Fig. 4). After selection for 4 weeks a $65 \%$ decrease of colony formation in cancer cells transfected with RASSF2 (mean $=4.6)$ compared to cells transfected with the empty vector $($ mean $=13.6 ; \mathrm{p}=0.019$, one-way
ANOVA test) was observed (Fig. 6A and 6B). Additionally, we analyzed the RASSF2 induced apoptosis in 1736 and FTC133 cells (Fig. 6C and 6D; additional file 4). Transfection of fluorescent RASSF2 caused a significantly increased apoptosis indicated by nuclei condensation/fragmentation compared to the fluorescent vector in 1736 and FTC133 ( $<0.001$ and $\mathrm{p}=0.07$, respectively). Apoptotic cells were exemplarily verified by TUNEL assay (Fig. S4). In FTC1736 the ratio of intact vs. apoptotic cells was for the vector: $96 \%$ vs. $4 \%$; for RASSF2wt: $88 \%$ vs. $12 \%$ and for RASSF2 $\triangle$ SARAH: $94 \%$ vs. 6\%, respectively. In FTC133 a similar trend was observed (Fig. 6D). For FTC133 the ratio of intact vs. apoptotic cells was the following for the vector: $75 \%$ vs. $25 \%$; for RASSF 2 wt: $62 \%$ vs. $38 \%$ and for RASSF $2 \Delta-$ SARAH: $78 \%$ vs. $22 \%$, respectively. Thus, RASSF $2 \Delta$ SARAH exhibited a significantly reduced rate of apoptotic cells in 1736 and FTC133 cells compared to wt RASSF2 ( $\mathrm{c}<0.05)$. In summary, our data suggest that RASSF2 tumor suppression function involves apoptotic regulation through binding of the proapoptotic kinases MST.

\section{Discussion}

Epigenetic inactivation of tumor suppressor genes is a fundamental event in the pathogenesis of malignant tumors. Here we report that RASSF2, a member of the 


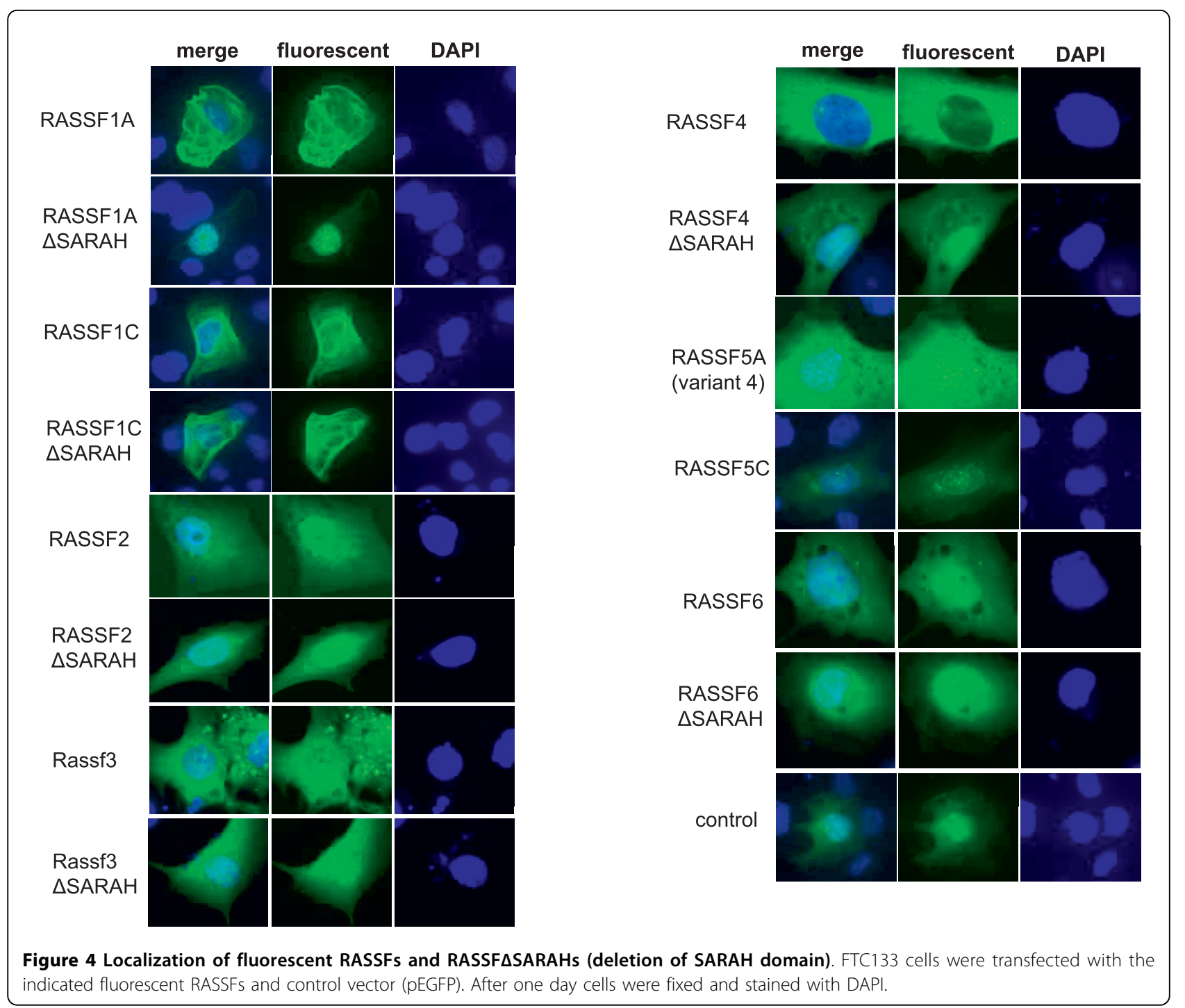

Ras Association Domain family, is frequently silenced in primary thyroid tumor and thyroid cancer cell lines. To the best of our knowledge this is the first report that shows that the epigenetic inactivation of RASSF2 is a common event in thyroid carcinogenesis. We also show that RASSF2 suppressed growth of thyroid cancer cells. The RASSF2 transcript can be detected in most normal tissues [20]. Down-regulation of RASSF2A by promoter hypermethylation has been shown in different tumor cell lines and primary tumors, including lung cancer, colon cancer, gastric cancer, nasopharyngeal carcinoma, head and neck cancer [20,26-33]. It has been reported that primary colorectal cancers, which showed KRAS or $B R A F$ mutations, also frequently showed RASSF2 methylation, and inactivation of RASSF2 enhanced KRASinduced oncogenic transformation $[28,30]$. RASSF2 binds directly to K-Ras in a GTP-dependent manner via the Ras effector domain [20]. In addition, patients with methylated $R A S S F 2 A$ promoter presented a higher frequency of lymph node metastasis [27]. Methylation of RASSF1A and RASSF2 was associated with poor outcome after radiotherapy in oral squamous cell carcinoma [34]. Here we report that the degree of RASSF2 methylation was associated with the age of thyroid cancer patients. It has been suggested that aberrant methylation of RASSF2 in plasma of colorectal cancer patients may serve as a cancer biomarker [35]. Thus, it will be interesting to determine hypermethylation of RASSF 2 in blood samples of thyroid cancer patients.

Regarding RASSF2's interaction with other RASSF members, it was shown to associate with RASSF3 and RASSF5 [29]. Our yeast two hybrid assay confirmed these bindings and additionally binding of RASSF1A and RASSF2 was observed. However these interactions were not observed in pulldown (Tab. 2). In general only the strong yeast two hybrid interactions could be 
Table 2 Summary of interaction analyses

\begin{tabular}{|c|c|c|c|}
\hline \multicolumn{2}{|c|}{ interaction partners } & \multirow{2}{*}{$\begin{array}{c}\begin{array}{c}\text { interaction in } \\
\text { Y2H }\end{array} \\
+/+\end{array}$} & \multirow{2}{*}{$\begin{array}{c}\begin{array}{c}\text { interaction in } \\
\text { pulldown }\end{array} \\
+ \text { na }\end{array}$} \\
\hline MST1/ & RASSF1A & & \\
\hline & RASSF2 & $+/+$ & $+/$ na \\
\hline & RASSF3 & $+/+$ & $+/$ na \\
\hline & RASSF4 & $+/+$ & $+/$ na \\
\hline & $\begin{array}{c}\text { RASSF5A } \\
\text { (V4) }\end{array}$ & $w / w$ & $-/ n a$ \\
\hline & RASSF5C & $+/+$ & $+/$ na \\
\hline & RASSF6 & $+/+$ & $+/$ na \\
\hline & WW45 & $+/+$ & $+/$ na \\
\hline \multirow[t]{6}{*}{ WW45 } & RASSF1A & w & - \\
\hline & RASSF2 & - & - \\
\hline & RASSF3 & w & - \\
\hline & RASSF4 & w & - \\
\hline & $\begin{array}{c}\text { RASSF5A } \\
(\text { V4) }\end{array}$ & w & na \\
\hline & RASSF6 & - & - \\
\hline \multirow[t]{6}{*}{ RASSF1A } & RASSF1A & + & - \\
\hline & RASSF2 & - & - \\
\hline & RASSF3 & - & - \\
\hline & RASSF4 & - & - \\
\hline & $\begin{array}{c}\text { RASSF5A } \\
\text { (V4) }\end{array}$ & - & - \\
\hline & RASSF6 & - & - \\
\hline \multirow[t]{6}{*}{ RASSF2 } & RASSF1A & w & - \\
\hline & RASSF2 & - & - \\
\hline & RASSF3 & + & - \\
\hline & RASSF4 & - & - \\
\hline & $\begin{array}{c}\text { RASSF5A } \\
(\text { V4) }\end{array}$ & - & - \\
\hline & RASSF6 & - & - \\
\hline \multirow[t]{6}{*}{ RASSF3 } & RASSF1A & - & - \\
\hline & RASSF2 & - & - \\
\hline & RASSF3 & w & w \\
\hline & RASSF4 & + & - \\
\hline & $\begin{array}{c}\text { RASSF5A } \\
\text { (V4) }\end{array}$ & - & na \\
\hline & RASSF6 & - & - \\
\hline \multirow[t]{6}{*}{ RASSF4 } & RASSF1A & - & - \\
\hline & RASSF2 & - & - \\
\hline & RASSF3 & + & - \\
\hline & RASSF4 & - & - \\
\hline & $\begin{array}{c}\text { RASSF5A } \\
\text { (V4) }\end{array}$ & - & - \\
\hline & RASSF6 & - & - \\
\hline \multirow[t]{6}{*}{ RASSF5C } & RASSF1A & + & $w$ \\
\hline & RASSF2 & + & - \\
\hline & RASSF3 & - & na \\
\hline & RASSF4 & w & - \\
\hline & $\begin{array}{c}\text { RASSF5A } \\
(\text { V4) }\end{array}$ & - & na \\
\hline & RASSF6 & - & \\
\hline
\end{tabular}

Table 2: Summary of interaction analyses (Continued)

\begin{tabular}{cccc}
\hline RASSF6 & RASSF1A & - & - \\
& RASSF2 & - & - \\
RASSF3 & - & - \\
RASSF4 & - & - \\
RASSF5A & - & - \\
(V4) & - & na \\
RASSF6 & - &
\end{tabular}

confirmed by pulldowns. Since yeast two hybrid assays are prone to false positive results, we rather agree with the pull down interactions. RASSF2 also binds both proapoptotic kinases MST1 and MST2 in yeast two hybrid assays and this was confirmed for RASSF2 and MST1 by pulldown. It has been suggested that binding of the classical RASSFs, MST1, MST2 and WW45 with each other is conducted by their SARAH domains [21]. Deletion of the SARAH domain of RASSF2 abolished binding with MST1 and MST2 [36,37]. It is interesting to note that all RASSFs and WW45 bind MST1, however only certain RASSFs (e.g. RASSF1A and RASSF5C) bind each other in pulldowns. This result suggests that SARAH domains show different binding properties.

RASSF2 exhibits several tumor suppressor properties, like inhibition of cell growth and induction of apoptosis $[20,27]$. Here we show that RASSF2 also acts as a novel tumor suppressor in thyroid carcinogenesis. It has been shown that RASSF2 binds and regulates the proapoptotic kinases MST1 and MST2 [36,37]. RASSF2 also engages the JNK pathway and induces apoptosis in an MST1-independent manner [37]. Other findings suggest that MST2 and RASSF2 form a complex, in which RASSF2 is maintained in a phosphorylated state by MST2 and protects MST2 from degradation and turnover [36]. However, the exact phosphorylation site of RASSF2 by MST2 was not revealed.

Additionally, we have found that RASSF5A is frequently hypermethylated in thyroid tissues, however methylation was not tumor specific since it was also observed in controls. Epigenetic silencing of RASSF5 in thyroid cancer has been analyzed previously, but was also not tumor specific [11,12]. Interestingly, RASSF5A and RASSF2 methylation was reduced in MTC compared to other cancers. It has been reported that RASSF5A expression is suppressed in FTC carrying PAX8-PPAR $\gamma$ fusions [11]. Methylation of the other investigated $\mathrm{CpG}$ island promoters of RAS effectors (RASSF3, RASSF4, MST1, MST2 and WW45) was uncommon.

\section{Conclusions}

In summary, we report frequent tumor-specific hypermethylation of RASSF 2 in thyroid cancer. Functional 


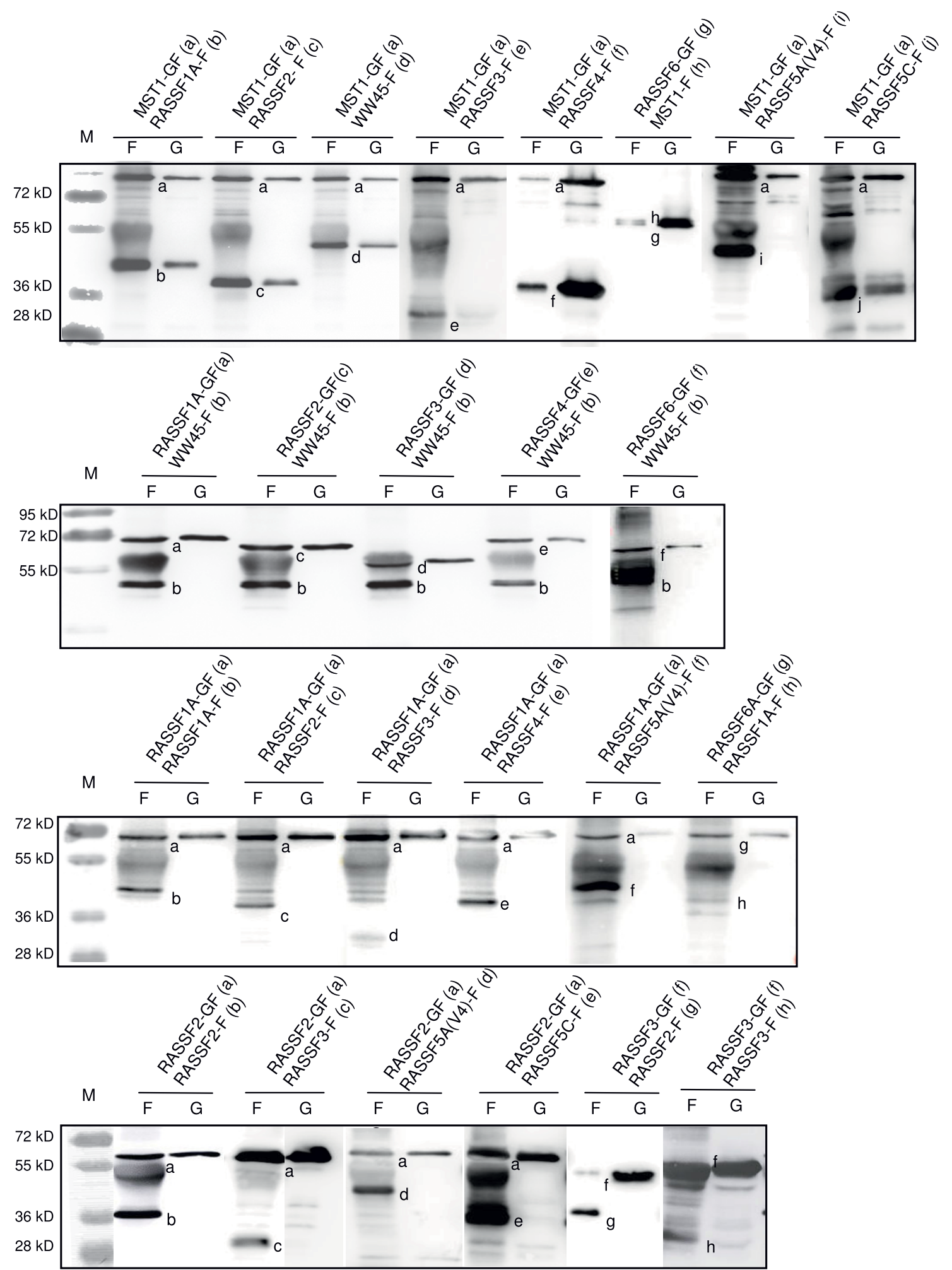

Figure 5 Binding studies of RASSFs, MST1 and WW45 in co-precipitation. Plasmids, that express the indicated GST-Flag-tagged-constructs (-GF) and Flag-tagged-constructs (-F) were transfected into HEK293T cells. After two days, total protein was extracted and Flag-tagged proteins were precipitated with anti-Flag-agarose (F) or glutathione sepharose $(G)$. Samples were separated on a 10\% PAGE gel and blotted. The precipitated and co-precipitated proteins were determined with anti-Flag-antibodies. The sizes of a protein marker (M) are indicated. 


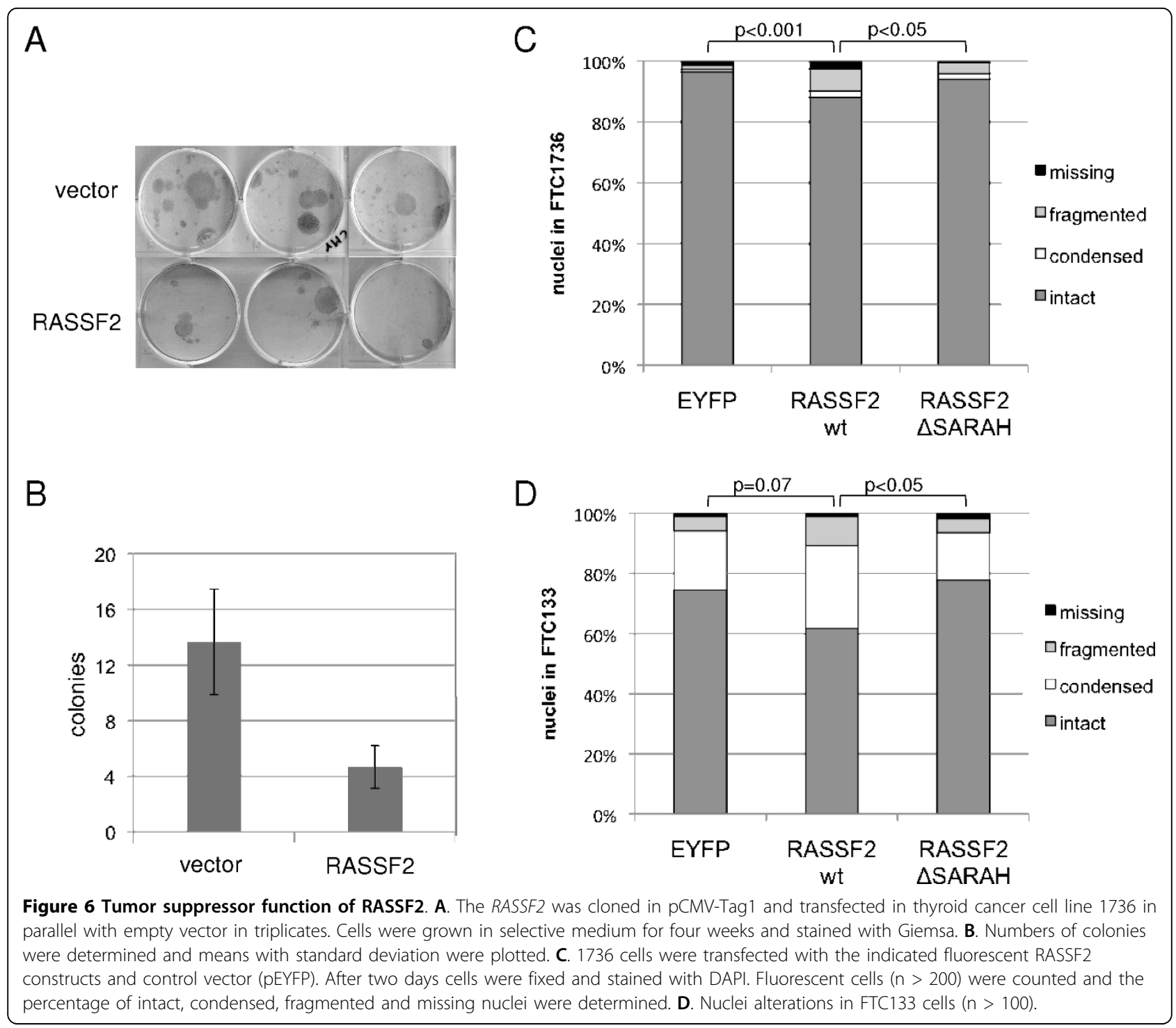

analysis showed that RASSF2 interacts with both proapoptotic kinases MST1 and MST2. Overexpression of RASSF2 induces apoptosis in thyroid cancer cell lines. These data suggest that RASSF2 acts as a proapoptotic tumor suppressor in thyroid carcinogenesis.

\section{Methods}

\section{Tissues and cell lines}

65 thyroid tissues, including 31 primary thyroid carcinomas (6 UTC, 3 MTC, 10 FTC, and 12 PTC), 10 follicular adenomas, 12 goiters and 12 normal thyroid samples were analyzed $[7,10]$. All patients signed informed consent at initial clinical investigation. The study was approved by local ethic committees. The mean age of the study population at the time of surgical resection was $51 \pm 15$ years and $40 \%$ of the patients were male. Nine human thyroid cancer cell lines: BcPaP (PTC),
FTC238, FTC236, FTC133, C643 (UTC), 8505C (UTC), 1736 (UTC), 8305C (UTC) and HTh74 (UTC) were cultured in the recommended medium and included in this study $[7,10]$. Genomic DNA was extracted from frozen tissues and cultured cells by a standard phenol/chloroform procedure.

\section{Methylation analysis}

Methylation of promoter regions (RASSF2, RASSF3, RASSF4, RASSF5A, RASSF5C, WW45, MST1 and MST2) was determined by COBRA and bisulfite sequencing [38,39]. Briefly, $100 \mathrm{ng}$ of bisulfite-treated DNA were amplified with $10 \mathrm{pmol}$ of primers in a reaction buffer containing $0.2 \mathrm{mM}$ dNTP mix, $1.5 \mathrm{mM} \mathrm{MgCl}, 10 \mathrm{pmol}$ of each primer and 1.5 U Taq polymerase (InViTek $\mathrm{GmBH}$, Berlin, Germany). For PCR primers and condition see additional file 5.20 to $50 \mathrm{ng}$ of PCR products 
were digested with $10 \mathrm{U}$ of TaqI or HpyCH4IV (NEB, Frankfurt, Germany) and analyzed on a $2 \%$ Tris-borate EDTA agarose gel. For bisulfite sequencing PCR products were cloned in the pGEM-T vector (Promega, Heidelberg, Germany) and sequenced (Genterprise, Mainz, Germany).

\section{Expression analysis}

Two thyroid cancer cell lines (1736 and FTC236) were treated for 4 days with $5 \mu \mathrm{M}$ or $10 \mu \mathrm{M}$ of 5 -aza-dC (Sigma, Taufkirchen, Germany). RNA was isolated using TRIzol-Reagent (Invitrogen, Karlsruhe, Germany). To eliminate DNA contamination $1 \mu \mathrm{g}$ RNA was incubated with $1 \mathrm{U}$ DNase I (Fermentas GmbH, St. Leon-Rot, Germany), $1 \mu \mathrm{l}$ 10X DNase I buffer and DEPC-treated water in $10 \mu \mathrm{l}$. After 15 minutes incubation at room temperature DNase was inactivated by adding $1 \mu \mathrm{l}$ of 25 $\mathrm{mM}$ EDTA and incubation at $65^{\circ} \mathrm{C}$ for $15 \mathrm{~min}$ and reversely transcribed using poly-dT primers and random hexamers in $20 \mu \mathrm{l}$ of RT-mix with MMLV reverse transcriptase (Promega, Heidelberg, Germany) for $1 \mathrm{~h}$ at $42^{\circ}$ C. Subsequently, $2 \mu \mathrm{l}$ of cDNA was amplified. For PCR primers and condition see additional file 6 .

\section{Statistical evaluation}

Statistical analysis was carried out using SPSS17 (SPSS, München, Germany). Categorical variables were plotted into contingency tables and evaluated using Fisher's exact test or one way ANOVA test. All reported $\mathrm{p}$-values are two-sided and considered significant for $\mathrm{p}<0.05$.

\section{Constructs}

The cDNAs of RASSF2, RASSF3, RASSF4, RASSF5A (variant 4), RASSF5C (variant 3), RASSF6, MST1, MST2 and WW45 were cloned after amplification of fragments from EST-clones DKFZp781O1747Q, IMAGp998A0813502Q, IRAKp961I1269Q, IRALp 962N0113Q (BC004270), IRAKp961L2026Q; DKFZp 686K23225Q, IRAKp961C0282Q, IRAKp961I0613Q and IRAKp961L0427Q (RZPD, Berlin, Germany), respectively. RASSF1A, RASSF1C and Rassf3 were described previously $[3,40]$. Mutant forms of constructs were generated with the QuickChange ${ }^{\circ}$ XL Site-Directed Mutagenesis Kit (Stratagene, La Jolla, USA) and appropriate primers. All constructs were confirmed by sequencing.

\section{Localization studies}

cDNAs were cloned into the fluorescence vector pEYFP (Clontech, Mountain View, USA). After transient transfection into FTC133 cells with HEKfectin (BioRad, München, Germany), the localization of fluorescent RASSF variants and the vector control were investigated with a fluorescence microscope (Axio Observer Z1 Zeiss, Jena, Germany). Nuclei of the cells were visualized by staining with DAPI $(0,1 \mu \mathrm{g} / \mathrm{ml}$ in PBS).

\section{Interaction studies using the yeast two-hybrid system}

The Matchmaker Two-hybrid system (Clontech, Mountain View, USA) was utilized. The yeast strain PJ69-4A was co-transformed with $0.5 \mu \mathrm{g}$ of each plasmid using the PEG/LiAc method. The interaction analysis was carried out on SD minimal medium plates without adenine and histidine and the transformation efficiency was determined on SD plates with adenine and histidine. The strength of interaction was investigated by quantification of the expression of the $\beta$-galactosidase reporter gene with o-nitrophenyl- $\beta$-D-galactopyranoside (ONPG) as substrate at OD $420 \mathrm{~nm}$.

\section{Interaction studies by co-precipitation}

cDNAs were cloned into the vector pCMV-Tag1 (Stratagene, La Jolla, USA) and/or in the modified vector pEBG (GST and FLAG tag). To investigate the interaction of specific RASSF forms, MST1, MST2 and WW45, co-transfections with HEKfectin (BioRad, München, Germany), were performed in HEK293T cells. Two days after transfection, total protein was extracted in FLAG-lysis buffer. The GST-fused proteins were precipitated with glutathione-sepharose (Amersham Biosciences, Freiburg, Germany). FLAG-tagged proteins were precipitated with anti-Flag-agarose (Sigma, Steinheim, Germany). Samples were separated on a 10\% PAGE gel and blotted. The interaction was determined with anti-FLAG-antibodies (Sigma, Steinheim, Germany).

\section{Proliferation assays}

RASSF2 was cloned into the vector pCMV-Tag1 (Stratagene, La Jolla, USA). The thyroid cancer cell line 1736 was transfected using HEKfectin (BioRad, München, Germany). Colonies were selected under $1 \mathrm{mg} / \mathrm{ml}$ Geneticin (Gibco, Karlsruhe, Germany) in DMEM for 4 weeks. Expression of RASSF2 was confirmed by RTPCR (data not shown).

\section{Apoptotic assays}

Thyroid cancer cells FTC133 and 1736 were seeded on glass slides and transfected with different RASSF2 constructs using HEKfectin (BioRad, München, Germany) and analyzed after two days of incubation. Therefore cells were fixed with paraformaldehyde, permeabilized with TritonX, DAPI stained and embedded in MOWIOL for fluorescent analysis. Transfected cells $(\mathrm{n}>100$ for FTC133 and $n>200$ for 1736) were analyzed for intact, condensed, fragmented or missing nucleus. 


\section{Additional material}

Additional file 1: Summary of bisulfite sequencing of RASSF2 and RASSF5A. Figure with the results of the bisulfite sequencing

Additional file 2: Quantitative interaction analysis in the ONPG assay. Graph of the quantitative yeast two-hybrid interaction results Additional file 3: Binding analysis of RASSF2 $\triangle$ SARAH and MST1 in co-precipitation. Figure of Western blot

Additional file 4: RASSF2 induced apoptosis. Figure of TUNEL assay. Additional file 5: Primer sequences and conditions for COBRA. Table of oligonucleotides used for methylation analysis

Additional file 6: Primer sequences and conditions for qRT-PCR.

Table of oligonucleotides used for expression analysis

\section{Abbreviations}

RASSF: Ras association domain family; MST: mammalian sterile 20-like kinase/ human Hippo homolog; WW45: human Salvador homolog; SARAH: SalvadorRASSF-Hippo; COBRA: combined bisulfite restriction analysis; 5-AZA-DC: 5aza-2'-deoxycytidine; UTC: undifferentiated thyroid carcinoma; PTC: papillary thyroid carcinoma; MTC: medullary thyroid carcinoma; FTC: follicular thyroid carcinoma
\end{abstract}

\section{Acknowledgements}

This work was supported by ATA THANC Research Grant to Undraga Schagdarsurengin. The research in Reinhard Dammann's laboratory is funded by Deutsche Krebshilfe and Deutsche Forschungsgemeinschaft (DFG DA552). These organizations had no involvement in the study design, in the acquisition, analysis and interpretation of data, in the writing of the manuscript, and in the decision to submit the manuscript for publication.

\section{Author details}

${ }^{1}$ Institute for Genetics, Justus-Liebig-University Giessen, Germany. ${ }^{2}$ Institute of Biochemistry, Medical Faculty, Justus-Liebig-University Giessen, Germany.

\section{Authors' contributions}

RHD has created the study. AMR, US, KS and RHD participated in the design of the study. AMR, US, CL, JH and KS acquired data. AMR, US, CL, JH and $\mathrm{RHD}$ controlled, analyzed and interpreted data. AMR, US, CL, JH and RHD prepared the manuscript. All authors read, corrected and approved the final manuscript.

\section{Competing interests}

The authors declare that they have no competing interests.

Received: 19 March 2010 Accepted: 29 September 2010 Published: 29 September 2010

\section{References}

1. Gimm O: Thyroid cancer. Cancer Lett 2001, 163:143-156.

2. Roman S, Mehta P, Sosa JA: Medullary thyroid cancer: early detection and novel treatments. Curr Opin Oncol 2009, 21:5-10.

3. Dammann R, Li C, Yoon JH, Chin PL, Bates S, Pfeifer GP: Epigenetic inactivation of a RAS association domain family protein from the lung tumour suppressor locus 3p21.3. Nat Genet 2000, 25:315-319.

4. Agathanggelou A, Cooper WN, Latif F: Role of the Ras-association domain family 1 tumor suppressor gene in human cancers. Cancer Res 2005, 65:3497-3508.

5. Dammann R, Schagdarsurengin U, Seidel C, Strunnikova M, Rastetter M Baier K, Pfeifer GP: The tumor suppressor RASSF1A in human carcinogenesis: an update. Histol Histopathol 2005, 20:645-663.

6. Hoque MO, Rosenbaum E, Westra WH, Xing M, Ladenson P, Zeiger MA, Sidransky D, Umbricht CB: Quantitative assessment of promoter methylation profiles in thyroid neoplasms. J Clin Endocrinol Metab 2005, 90:4011-4018
7. Schagdarsurengin U, Gimm O, Hoang-Vu C, Dralle H, Pfeifer GP, Dammann R: Frequent epigenetic silencing of the $\mathrm{CpG}$ island promoter of RASSF1A in thyroid carcinoma. Cancer Res 2002, 62:3698-3701.

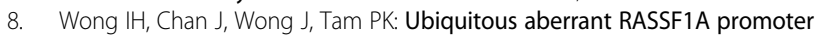
methylation in childhood neoplasia. Clin Cancer Res 2004, 10:994-1002.

9. Xing M, Cohen Y, Mambo E, Tallini G, Udelsman R, Ladenson PW, Sidransky D: Early occurrence of RASSF1A hypermethylation and its mutual exclusion with BRAF mutation in thyroid tumorigenesis. Cancer Res 2004, 64:1664-1668.

10. Schagdarsurengin U, Gimm O, Dralle H, Hoang-Vu C, Dammann R: CpG island methylation of tumor-related promoters occurs preferentially in undifferentiated carcinoma. Thyroid 2006, 16:633-642.

11. Foukakis T, Au AY, Wallin G, Geli J, Forsberg L, Clifton-Bligh R, Robinson BG, Lui WO, Zedenius J, Larsson C: The Ras effector NORE1A is suppressed in follicular thyroid carcinomas with a PAX8-PPARgamma fusion. J Clin Endocrinol Metab 2006, 91:1143-1149.

12. Nakamura N, Carney JA, Jin L, Kajita S, Pallares J, Zhang H, Qian X, Sebo TJ, Erickson LA, Lloyd RV: RASSF1A and NORE1A methylation and BRAFV600E mutations in thyroid tumors. Lab Invest 2005, 85:1065-1075.

13. Richter AM, Pfeifer GP, Dammann RH: The RASSF proteins in cancer; from epigenetic silencing to functional characterization. Biochim Biophys Acta 2009, 1796:114-128.

14. Sherwood V, Manbodh R, Sheppard C, Chalmers AD: RASSF7 is a member of a new family of RAS association domain-containing proteins and is required for completing mitosis. Mol Biol Cell 2008, 19:1772-1782.

15. Sherwood V, Recino A, Jeffries A, Ward A, Chalmers AD: The N-terminal RASSF family: a new group of Ras-association-domain-containing proteins, with emerging links to cancer formation. Biochem J 2010, 425:303-311.

16. van der Weyden $L$, Adams DJ: The Ras-association domain family (RASSF) members and their role in human tumourigenesis. Biochim Biophys Acta 2007, 1776:58-85.

17. Schagdarsurengin U, Richter AM, Wohler C, Dammann RH: Frequent epigenetic inactivation of RASSF10 in thyroid cancer. Epigenetics 2009, 4:571-576.

18. Allen NP, Donninger H, Vos MD, Eckfeld K, Hesson L, Gordon L, Birrer MJ, Latif F, Clark GJ: RASSF6 is a novel member of the RASSF family of tumor suppressors. Oncogene 2007, 26:6203-6211.

19. Khokhlatchev A, Rabizadeh S, Xavier R, Nedwidek M, Chen T, Zhang XF, Seed B, Avruch J: Identification of a novel Ras-regulated proapoptotic pathway. Curr Biol 2002, 12:253-265.

20. Vos MD, Ellis CA, Elam C, Ulku AS, Taylor BJ, Clark GJ: RASSF2 is a novel KRas-specific effector and potential tumor suppressor. J Biol Chem 2003, 278:28045-28051.

21. Scheel $H$, Hofmann $K$ : A novel interaction motif, SARAH, connects three classes of tumor suppressor. Curr Biol 2003, 13:R899-900.

22. Ortiz-Vega S, Khokhlatchev A, Nedwidek M, Zhang XF, Dammann R, Pfeifer GP, Avruch J: The putative tumor suppressor RASSF1A homodimerizes and heterodimerizes with the Ras-GTP binding protein Nore1. Oncogene 2002, 21:1381-1390.

23. Chan EH, Nousiainen M, Chalamalasetty RB, Schafer A, Nigg EA, Sillje HH: The Ste20-like kinase Mst2 activates the human large tumor suppressor kinase Lats1. Oncogene 2005, 24:2076-2086.

24. Hwang E, Ryu KS, Paakkonen K, Guntert P, Cheong HK, Lim DS, Lee JO, Jeon $\mathrm{YH}$, Cheong $\mathrm{C}$ : Structural insight into dimeric interaction of the SARAH domains from Mst1 and RASSF family proteins in the apoptosis pathway. Proc Natl Acad Sci USA 2007, 104:9236-9241.

25. Udan RS, Kango-Singh M, Nolo R, Tao C, Halder G: Hippo promotes proliferation arrest and apoptosis in the Salvador/Warts pathway. Nat Cell Biol 2003, 5:914-920.

26. Endoh M, Tamura G, Honda T, Homma N, Terashima M, Nishizuka S, Motoyama T: RASSF2, a potential tumour suppressor, is silenced by CpG island hypermethylation in gastric cancer. Br J Cancer 2005, 93:1395-1399.

27. Zhang Z, Sun D, Van do N, Tang A, Hu L, Huang G: Inactivation of RASSF2A by promoter methylation correlates with lymph node metastasis in nasopharyngeal carcinoma. Int J Cancer 2007, 120:32-38.

28. Akino K, Toyota M, Suzuki H, Mita H, Sasaki Y, Ohe-Toyota M, Issa JP, Hinoda Y, Imai K, Tokino T: The Ras effector RASSF2 is a novel tumor-suppressor gene in human colorectal cancer. Gastroenterology 2005, 129:156-169. 
29. Hesson LB, Wilson R, Morton D, Adams C, Walker M, Maher ER, Latif F: CpG island promoter hypermethylation of a novel Ras-effector gene RASSF2A is an early event in colon carcinogenesis and correlates inversely with Kras mutations. Oncogene 2005, 24:3987-3994.

30. Park HW, Kang HC, Kim IJ, Jang SG, Kim K, Yoon HJ, Jeong SY, Park JG: Correlation between hypermethylation of the RASSF2A promoter and $\mathrm{K}$ ras/BRAF mutations in microsatellite-stable colorectal cancers. Int J Cancer 2007, 120:7-12.

31. Steinmann K, Sandner A, Schagdarsurengin U, Dammann RH: Frequent promoter hypermethylation of tumor-related genes in head and neck squamous cell carcinoma. Oncol Rep 2009, 22:1519-1526.

32. Kaira K, Sunaga N, Tomizawa $Y$, Yanagitani $N$, Ishizuka T, Saito R, Nakajima $T$, Mori M: Epigenetic inactivation of the RAS-effector gene RASSF2 in lung cancers. Int J Oncol 2007, 31:169-173.

33. Cooper WN, Dickinson RE, Dallol A, Grigorieva EV, Pavlova TV, Hesson LB, Bieche I, Broggini M, Maher ER, Zabarovsky ER, et al: Epigenetic regulation of the ras effector/tumour suppressor RASSF2 in breast and lung cancer. Oncogene 2008, 27:1805-1811.

34. Huang KH, Huang SF, Chen IH, Liao CT, Wang HM, Hsieh LL: Methylation of RASSF1A, RASSF2A, and HIN-1 is associated with poor outcome after radiotherapy, but not surgery, in oral squamous cell carcinoma. Clin Cancer Res 2009, 15:4174-4180.

35. Lee BB, Lee EJ, Jung EH, Chun HK, Chang DK, Song SY, Park J, Kim DH: Aberrant methylation of APC, MGMT, RASSF2A, and Wif-1 genes in plasma as a biomarker for early detection of colorectal cancer. Clin Cancer Res 2009, 15:6185-6191.

36. Cooper WN, Hesson LB, Matallanas D, Dallol A, von Kriegsheim A, Ward R, Kolch W, Latif F: RASSF2 associates with and stabilizes the proapoptotic kinase MST2. Oncogene 2009, 28:2988-2998.

37. Song H, Oh S, Oh HJ, Lim DS: Role of the tumor suppressor RASSF2 in regulation of MST1 kinase activity. Biochem Biophys Res Commun 2010, 391:969-973.

38. Dammann R, Yang G, Pfeifer GP: Hypermethylation of the cpG island of Ras association domain family $1 \mathrm{~A}$ (RASSF1A), a putative tumor suppressor gene from the 3 p21.3 locus, occurs in a large percentage of human breast cancers. Cancer Res 2001, 61:3105-3109.

39. Xiong Z, Laird PW: COBRA: a sensitive and quantitative DNA methylation assay. Nucleic Acids Res 1997, 25:2532-2534.

40. Tommasi S, Dammann R, Jin SG, Zhang Xf XF, Avruch J, Pfeifer GP: RASSF3 and NORE1: identification and cloning of two human homologues of the putative tumor suppressor gene RASSF1. Oncogene 2002, 21:2713-2720.

doi:10.1186/1476-4598-9-264

Cite this article as: Schagdarsurengin et al: Frequent epigenetic inactivation of RASSF2 in thyroid cancer and functional consequences. Molecular Cancer 2010 9:264.

\section{Submit your next manuscript to BioMed Central and take full advantage of:}

- Convenient online submission

- Thorough peer review

- No space constraints or color figure charges

- Immediate publication on acceptance

- Inclusion in PubMed, CAS, Scopus and Google Scholar

- Research which is freely available for redistribution

Submit your manuscript at www.biomedcentral.com/submit
Biomed Central 